\title{
OSCILLATION CRITERIA FOR HAMILTONIAN MATRIX DIFFERENCE SYSTEMS
}

\section{H. ERBE AND PENGXIANG YAN}

(Communicated by Hal L. Smith)

Abstract. We obtain some oscillation criteria for the Hamiltonian difference system

$$
\left\{\begin{array}{l}
\Delta Y(t)=B(t) Y(t+1)+C(t) Z(t), \\
\Delta Z(t)=-A(t) Y(t+1)-B^{*}(t) Z(t),
\end{array}\right.
$$

where $A, B, C, Y, Z$ are $d \times d$ matrix functions. As a corollary, we establish the validity of an earlier conjecture for a second-order matrix difference system.

\section{INTRODUCTION AND PRELIMINARY RESULTS}

Consider the linear Hamiltonian difference system

$$
\begin{aligned}
& \Delta y(t)=B(t) y(t+1)+C(t) z(t), \\
& \Delta z(t)=-A(t) y(t+1)-B^{*}(t) z(t),
\end{aligned}
$$

the corresponding matrix system

$$
\begin{aligned}
& \Delta Y(t)=B(t) Y(t+1)+C(t) Z(t), \\
& \Delta Z(t)=-A(t) Y(t+1)-B^{*}(t) Z(t),
\end{aligned}
$$

and the Riccati equation

$$
\begin{aligned}
& \Delta W(t)+A(t)+B^{*}(t) W(t)+W(t) B(t)-B^{*}(t) W(t) B(t) \\
& \quad+(I-B(t))^{*} W(t)\left(C^{-1}(t)+W(t)\right)^{-1} W(t)(I-B(t))=0,
\end{aligned}
$$

where $A(t), B(t), C(t), W(t), Y(t), Z(t)$ are $d \times d$ matrices with $A(t), C(t)$ Hermitian, $C(t)>0$, and $I-B(t)$ invertible. Here $y(t), z(t)$ are $d \times 1$ vectors and $t$ takes on integer values in $[M-1, N+1]$, where $M, N$ are two integers.

In $[4,5]$ the authors extended many of the results to equations $(1.1)-(1.3)$ which had been developed for linear Hamiltonian differential systems of the form

$$
\begin{aligned}
& y^{\prime}(x)=B(x) y(x)+C(x) z(x), \\
& z^{\prime}(x)=-A(x) y(x)-B^{*}(x) z(x) .
\end{aligned}
$$

Received by the editors February 24, 1992.

1991 Mathematics Subject Classification. Primary 39A10.

Key words and phrases. Disconjugacy, difference system, Riccati system.

Research was supported by NSERC-Canada. 
Here $x \in I$ is a finite or infinite interval, $A, B, C$ are continuous $d \times d$ matrix-valued functions, and $y, z$ are $d \times 1$ vector functions. Many of the results for (1.4) may be found in the book of Coppel [2], and in [4, 5] it was shown that discrete analogues of many of these results may be obtained. Related work on symmetric three-term recurrences may be found in [1] and the references therein. In this paper, we shall obtain some oscillation and disconjugacy criterion for (1.1), (1.2) and, as a consequence of our results, shall prove a generalization of a conjecture of Peterson and Ridenhour [7]. We recall some notation and definitions.

We say (1.1) is disconjugate on $[M-1, N+1]$ iff for any nontrivial prepared solution $\{y(t), z(t)\}$ of $(1.1)$ there exists at most one integer $p \in[M-1, N]$ such that either $y^{*}(p) C^{-1}(p)(I-B(p)) y(p+1) \leq 0$ when $y(p) \neq 0$ or $y(p)=0$. Recall that a solution $\{y(t), z(t)\}$ of $(1.1)$ is said to be prepared if $y^{*}(t) z(t)$ is real valued and that a solution $\{Y(t), Z(t)\}$ of (1.2) is said to be prepared if $Y^{*}(t) Z(t)$ is Hermitian. We say a prepared solution of (1.2) is a conjoined basis if $\operatorname{Rank}\left[\begin{array}{l}Y(t) \\ Z(t)\end{array}\right] \equiv d$, and it is said to be recessive at $\infty$ if there exists an integer $M_{0}$ for which

$$
Y^{*}(t) C^{-1}(t)(I-B(t)) Y(t+1)>0, \quad t \geq M_{0},
$$

and

$$
\lim _{n \rightarrow \infty} \sum_{s=M_{0}}^{n} u^{*}\left(Y^{*}(s) C^{-1}(s)(I-B(s)) Y(s+1)\right)^{-1} u=\infty
$$

for every unit vector $u$. A prepared solution of (1.2) is said to be dominant at $\infty$ if (1.5) holds for some integer $M_{0}$ and

$$
\sum_{s=M_{0}}^{\infty} u^{*}\left(Y^{*}(s) C^{-1}(s)(I-B(s)) Y(s+1)\right)^{-1} u
$$

converges for every unit vector $u$.

Equation (1.1) is said to be eventually disconjugate in case there exists an integer $M_{0}$ such that $(1.1)$ is disconjugate on $\left[M_{0}-1, N_{1}+1\right]$ for all integers $N_{1}>M_{0}$.

We introduce the following quadratic forms:

$$
q[u]=\sum_{t=M}^{N+1}\left(z^{*}(t-1) C(t-1) z(t-1)-y^{*}(t) A(t-1) y(t)\right),
$$

where

$$
\begin{gathered}
u=\{y(t), z(t)\} \in \Omega=\left\{y, z \in C^{d}: y(M-1)=0=y(N+1),\right. \\
\Delta y(t)=B(t) y(t+1)+C(t) z(t)\},
\end{gathered}
$$

and

$$
Q[U]=\sum_{t=M}^{N+1}\left(Z^{*}(t-1) C(t-1) Z(t-1)-Y^{*}(t) A(t-1) Y(t)\right),
$$

where

$$
\begin{gathered}
U=\{Y(t), Z(t)\} \in \Lambda=\left\{Y, Z \in C^{d \times d}: Y(M-1)=0=Y(N+1),\right. \\
\Delta Y(t)=B(t) Y(t+1)+C(t) Z(t)\} .
\end{gathered}
$$


We introduce the further notation:

$\Lambda^{+}:=\left\{U \in \Lambda:\right.$ there is a $t_{0}, M-1 \leq t_{0} \leq N-1$, such that $Y\left(t_{0}\right)=0$ and $Y\left(t_{0}+1\right)$ is nonsingular or there is $M+1 \leq t_{0} \leq N+1$ such that $Y\left(t_{0}\right)=0$ and $Y\left(t_{0}-1\right)$ is nonsingular .

We say $q$ is positive on $\Omega$ provided $q[u] \geq 0$ for all $u \in \Omega$ and $q=0$ iff $u \equiv 0 ; Q$ is positive definite on $\Lambda$ provided, for all $U \in \Lambda, Q[U] \geq 0$ and $Q=0$ iff $U \equiv 0 ; Q$ is strictly positive on $\Lambda^{+}$if $Q[U]>0$ for all $U \in \Lambda^{+}$.

The following results were established in $[4,5]$ : The first theorem may be regarded as a discrete version of the "Reid Roundabout Theorem" (cf. Ahlbrandt [1]).

Theorem 1. The following are equivalent:

(i) Equation (1.1) is disconjugate on $[M-1, N+1]$.

(ii) $q[u]$ is positive definite on $\Omega$.

(iii) $Q[U]$ is positive definite on $\Lambda$ and strictly positive on $\Lambda^{+}$.

(iv) There exists a Hermitian solution of the Riccati equation (3) such that $C^{-1}(t)+W(t)>0, t \in[M-1, N]$.

(v) There exists a solution of equation (1.2) such that

$$
Y^{*}(t) C^{-1}(t)(I-B(t)) Y(t+1)>0, \quad t \in[M-1, N] .
$$

Theorem 2. Assume (1.1) is eventually disconjugate. Then it follows that:

(i) every conjoined basis $\{Y(t), Z(t)\}$ satisfies

$$
Y^{*}(t) C^{-1}(t)(I-B(t)) Y(t+1)>0, \quad t \geq M_{1} \geq M\left(M_{1} \text { large enough }\right) ;
$$

(ii) there exists a solution $\eta_{0}=\left\{Y_{0}(t), Z_{0}(t)\right\}$ of (1.2) which is recessive at $\infty$;

(iii) if $\eta_{1}=\left\{Y_{1}(t), Z_{1}(t)\right\}$ is any prepared solution of (1.2) such that $Z_{0}^{*}(t) Y_{1}(t)-Y_{0}^{*}(t) Z_{1}(t)$ is invertible, then $\eta_{1}$ is a dominant solution of (1.2) and $Y_{1}^{-1}(t) Y_{0}(t) \rightarrow 0$ (zero matrix) as $t \rightarrow \infty$.

By using Theorem 1, one can obtain a comparison theorem between the two systems:

$$
\begin{aligned}
& \Delta y(t)=B_{i}(t) y(t+1)+C_{i}(t) z(t), \\
& \Delta z(t)=-A_{i}(t) y(t+1)-B_{i}(t) z(t),
\end{aligned}
$$

where we make the assumption on $(1.8)_{i}$ as (1.1)

Denote

$$
D_{i}(t)=\left[\begin{array}{cc}
C_{i}^{-1}(t) & -B_{i}^{*} C_{i}^{-1}(t) \\
-C_{i}^{-1}(t) B_{i}(t) & B_{i}^{*}(t) C_{i}^{-1}(t) B_{i}(t)-A_{i}(t)
\end{array}\right], \quad i=1,2
$$

The following is then a generalized Sturm Comparison Theorem.

Theorem 3. If $(1.8)_{1}$ is disconjugate on $[M-1, N+1]$ and $D_{2}(t) \geq D_{1}(t)$ on $[M-1, N+1]$, then $(1.8)_{2}$ is disconjugate also. 
Proof. For $u_{1}=\left\{y_{1}(t), z_{1}(t)\right\}$ with $y_{1}(M-1)=0=y_{1}(N+1)$ and $\Delta y_{1}(t)=$ $B_{1}(t) y_{1}(t+1)+C_{1}(t) z_{1}(t)$, i.e., $z_{1}(t)=C_{1}^{-1}(t)\left(\Delta y_{1}(t)-B_{1}(t) y_{1}(t+1)\right)$, we have

$$
\begin{aligned}
q_{1}\left[u_{1}\right] & =\sum_{t=M-1}^{N}\left(z_{1}^{*}(t) C_{1}(t) z_{1}(t)-y_{1}^{*}(t+1) A_{1}(t) y_{1}(t+1)\right) \\
& =\sum_{t=M-1}^{N}\left(\Delta y_{1}^{*}(t), y_{1}^{*}(t+1)\right) D_{1}(t)\left(\begin{array}{c}
\Delta y_{1}(t) \\
y_{1}(t+1)
\end{array}\right),
\end{aligned}
$$

and for $u_{2}=\left\{y_{1}(t), z_{2}(t)\right\}$ with $z_{2}(t)=C_{2}^{-1}(t)\left(\Delta y-1(t)-B_{2}(t) y_{1}(t)\right)$, we have

$$
\begin{aligned}
q_{2}\left[u_{2}\right] & =\sum_{t=M-1}^{N}\left(\Delta y_{1}^{*}(t), y_{1}^{*}(t+1)\right) D_{2}(t)\left(\begin{array}{c}
\Delta y_{1}(t) \\
y_{1}(t+1)
\end{array}\right) \\
& \geq \sum_{t=M-1}^{N}\left(\Delta y_{1}^{*}(t), y_{1}^{*}(t+1)\right) D_{1}(t)\left(\begin{array}{c}
\Delta y_{1}(t) \\
y_{1}(t+1)
\end{array}\right)=q_{1}\left[u_{1}\right] .
\end{aligned}
$$

By Theorem 1, we know $q_{1}\left[u_{1}\right] \geq 0$ and so $q_{2}\left[u_{2}\right] \geq 0$, i.e., $(1.8)_{2}$ is disconjugate.

Corollary 4. If $(1.8)_{1}$ is disconjugate and $B_{1}(t)=B_{2}(t), A_{1}(t) \geq A_{2}(t), C_{1}(t) \geq$ $C_{2}(t)$, then $(1.8)_{2}$ is disconjugate.

Proof. This can be shown from Theorem 3 and

$$
D_{i}(t)=\left(\begin{array}{cc}
I & 0 \\
-B_{i}^{*}(t) & I
\end{array}\right)\left(\begin{array}{cc}
C_{i}^{-1}(t) & 0 \\
0 & -A_{i}(t)
\end{array}\right)\left(\begin{array}{cc}
I & -B_{i}(t) \\
0 & i
\end{array}\right) .
$$

Matrix systems. Next we consider the oscillation of solutions of the matrix system (1.2).

Definition. The Hamiltonian matrix difference system $(1.2)$ is said to be nonoscillatory if for each conjoined basis $\{Y(t), Z(t)\}$ there exists an integer $t_{0}$ such that, for $t \geq t_{0} \geq M-1$, we have

$$
Y^{*}(t) C^{-1}(t)(I-B(t)) Y(t+1)>0 .
$$

Otherwise we say it is oscillatory.

From Theorem 1, we know if (1.2) is nonoscillatory, then (1.1) is eventually disconjugate. If we suppose that there is a Hermitian solution $W(t)$ of (1.3) with $W(t)+C^{-1}(t)>0$, then this solution satisfies the rewritten Riccati equation (1.3):

$$
\Delta W(t)+G^{*}(t) G(t)+h(t)=0
$$

or

$$
\Delta W(t)+\rho(t)+h(t)=0
$$


where

$$
\begin{aligned}
G(t) & =\left(C^{-1}(t)+W(t)\right)^{-1 / 2} W(t)(I-B(t))+\left(C^{-1}(t)+W(t)\right)^{1 / 2} B(t), \\
h(t) & =A(t)-B^{*}(t) C^{-1}(t) B(t) \\
\rho(t) & =\left(W(t)+C^{-1}(t) B(t)\right)^{*}\left(C^{-1}(t)+W(t)\right)^{-1}\left(W(t)+C^{-1}(t) B(t)\right) .
\end{aligned}
$$

Denote by $F$ the set of all sequences of real numbers $s=\{s(t)\}_{t=0}^{\infty}$ with $0 \leq s(t) \leq 1$ and $\sum_{\tau=0}^{\infty} s(\tau)=+\infty$.

Let $S(t)=\sum_{\tau=0}^{t} s(\tau), S\left(t, t_{0}\right)=\sum_{\tau=t_{0}}^{t} s(\tau), l(t)=\lambda_{d}\left(C^{-1}(t)\right)$, and $L(t)=$ $\lambda_{1}\left(C^{-1}(t)\right)$. Here we suppose that the eigenvalues of $C^{-1}(t)$ are ordered with

$$
\lambda_{1}\left(C^{-1}(t)\right) \geq \lambda_{2}\left(C^{-1}(t)\right) \geq \cdots \geq \lambda_{d}\left(C^{-1}(t)\right) .
$$

We introduce the following conditions which will be used in subsequent results:

$\left(S_{1}\right) \quad \lim \sup _{t \rightarrow \infty} S^{-1-\alpha}(t) \sum_{\tau=0}^{t} S(\tau) L(\tau+1)<+\infty$;

$\left(S_{2}\right) \quad \lim \sup _{t \rightarrow \infty} S^{-\alpha}(t) L(t)<+\infty$; where $\alpha \geq 0$.

Similar to [3], we can prove

Theorem 5. Let $\left(S_{1}\right)$ hold for some $s \in F$. Then equation (1.2) is oscillatory provided

$$
\limsup _{t \rightarrow \infty} S^{-1-\alpha}(t) \lambda_{1}\left(\sum_{\tau=0}^{t} S(\tau) \sum_{k=0}^{\tau}\left(A(k)-B^{*}(k) C^{-1}(k) B(k)\right)\right)=+\infty
$$

Theorem 6. Let $\left(S_{2}\right)$ hold for some $s \in F$. Then (1.2) is oscillatory provided

$$
\limsup _{t \rightarrow \infty} S^{-1-\alpha}(t) \lambda_{1}\left(\sum_{\tau=0}^{t}\left(A(\tau)-B^{*}(\tau) C^{-1}(\tau) B(\tau)\right)\right)=+\infty .
$$

Example. Let

$$
C(t)=\left(\begin{array}{cc}
t^{-\alpha-1 / 2} & 0 \\
0 & t^{-\alpha}
\end{array}\right), \quad A(t)=\left(\begin{array}{cc}
t^{\alpha+1 / 2} & \frac{1}{2} \\
\frac{1}{2} & t^{\alpha}
\end{array}\right),
$$

$a \geq 0, B(t)=\frac{1}{2} I$. From Theorem 6 it follows that (1.2) is oscillatory in this case.

Theorem 7. If (1.2) is nonoscillatory, then there exists $t_{0}$ such that, for all $t \geq t_{0}$, we have

$$
\begin{aligned}
A\left(t_{0}\right) & +\sum_{\tau=t_{0}+1}\left(A(\tau)-B^{*}(\tau) C^{-1}(\tau) B(\tau)\right) \\
& <C^{-1}(t+1)+\left(I-B\left(t_{0}\right)\right)^{*} C^{-1}\left(t_{0}\right)\left(I-B\left(t_{0}\right)\right) .
\end{aligned}
$$

Proof. Since (1.2) is nonoscillatory, there exists a sufficiently large integer $t_{0}$ and a Hermitian matrix solution of (1.11) with $C^{-1}(t)+W(t)>0$ for $t \geq t_{0}$. 
Taking the summation of both sides of (1.11) from $t_{0}$ to $t$, we obtain

$$
\begin{aligned}
&-W(t+1)= \sum_{\tau=t_{0}+1}^{t} h(\tau)+\sum_{\tau=t_{0}+1}^{t} \rho(\tau)+A\left(t_{0}\right)-W\left(t_{0}\right)+W\left(t_{0}\right) B\left(t_{0}\right) \\
&+B^{*}\left(t_{0}\right) W\left(t_{0}\right)-B^{*}\left(t_{0}\right) W\left(t_{0}\right) B\left(t_{0}\right)+\left(I-B\left(t_{0}\right)\right)^{*} W\left(t_{0}\right) \\
& \times\left(C^{-1}\left(t_{0}\right)+W\left(t_{0}\right)\right)^{-1} W\left(t_{0}\right)\left(I-B\left(t_{0}\right)\right) \\
& \geq \sum_{\tau=t_{0}+1}^{t} h(\tau)+A\left(t_{0}\right) \\
&+\left(I-B\left(t_{0}\right)\right)^{*}\left(W\left(t_{0}\right)\left(C^{-1}+W\left(t_{0}\right)\right)^{-1} W\left(t_{0}\right)-W\left(t_{0}\right)\right)\left(I-B\left(t_{0}\right)\right) \\
&= \sum_{\tau=t_{0}+1}^{t} h(\tau)+A\left(t_{0}\right) \\
&-\left(I-B\left(t_{0}\right)\right)^{*} C^{-1}\left(t_{0}\right)\left(C^{-1}\left(t_{0}\right)+W\left(t_{0}\right)\right)^{-1} W\left(t_{0}\right)\left(I-B\left(t_{0}\right)\right) \\
&= \sum_{\tau=t_{0}+1}^{t} h(\tau)+A\left(t_{0}\right)-\left(I-B\left(t_{0}\right)\right)^{*} C^{-1}\left(t_{0}\right)\left(I-B\left(t_{0}\right)\right) \\
&+\left(I-B\left(t_{0}\right)\right)^{*}\left(C^{-1}\left(t_{0}\right)-C^{-1}\left(t_{0}\right)\right. \\
&\left.\quad \times\left(C^{-1}\left(t_{0}\right)+w\left(t_{0}\right)\right)^{-1} W\left(t_{0}\right)\right)\left(I-B\left(t_{0}\right)\right) \\
&> \sum_{\tau=t_{0}+1}^{t} h(\tau)+A\left(t_{0}\right)-\left(I-B\left(t_{0}\right)\right)^{*} C^{-1}\left(t_{0}\right)\left(I-B\left(t_{0}\right)\right) . \\
&+\left(I-B\left(t_{0}\right)\right)^{*} C^{-1}\left(t_{0}\right)\left(C^{-1}\left(t_{0}\right)+W\left(t_{0}\right)\right)^{-1} C^{-1}\left(t_{0}\right)\left(I-B\left(t_{0}\right)\right) \\
& \sum_{\tau=t_{0}+1}^{t} h(\tau)+A\left(t_{0}\right)-\left(I-B\left(t_{0}\right)\right)^{*} C^{-1}\left(t_{0}\right)\left(I-B\left(t_{0}\right)\right) \\
&(I-I)
\end{aligned}
$$

From $-W(t+1)<C^{-1}(t+1)$, the result follows.

Note. Taking $t=t_{0}$, we get [5, Proposition 2.1]. If $B(t)=0, C(t) \equiv I$, we get [7, Theorem 1].

From [4] we may express $Q[U]$ in the equivalent form

$$
Q[U]=\left.Y^{*}(t) W(t) Y(t)\right|_{M-1} ^{N+1}+\sum_{t=M-1}^{N} F^{*}(t) F(t)
$$

where

$$
\begin{aligned}
& F(t)=\left(C^{-1}(t)+W(t)\right)^{-1 / 2} W(t)(I-B(t)) Y(t+1)-\left(C^{-1}(t)+W(t)\right)^{1 / 2} C(t) Z(t), \\
& U=\{Y(t), Z(t)\} \in \Lambda, \text { and (1.2) is nonoscillatory. }
\end{aligned}
$$

Theorem 8. Suppose $C(t) \equiv I, B^{*}(t)+B(t) \leq B^{*}(t) B(t)$, and there exists $U=\{Y(t), Z(t)\}$ with $\Delta Y(t)=B(t) Y(t+1)+Z(t)$ such that

$$
\limsup _{N \rightarrow \infty} \lambda_{1}(Q[U])=-\infty \text {. }
$$

Then (1.2) is oscillatory.

Note. If $B(t) \equiv 0$, then this is [7, Theorem 5]. 
Proof. Suppose not, i.e., suppose (1.2) is nonoscillatory. Then by Theorem 2 there exists a solution $\{Y(t), Z(t)\}$ of $(1.2)$ and an integer $t_{0}$ such that

$$
\sum_{t=t_{0}}^{\infty}\left(Y^{*}(t)(I-B(t)) Y(t+1)\right)^{-1}=\tau \quad(\text { constant matrix). }
$$

We are going to prove that $Y^{*}(t)(I-B(t)) Y(t+1)$ is decreasing. To see this, observe that

(1.15)

$$
\begin{gathered}
\Delta\left(Y^{*}(t)(I-B(t)) Y(t+1)\right) \\
=Y^{*}(t+1)(\Delta Y(t)+\Delta Z(t))+\Delta Y^{*}(t)(I-B(t)) Y(t+1) \\
=Y^{*}(t+1)\left[I-(I+W(t))^{-1}(I-B(t))+W(t+1)\right. \\
-W(t)(I+W(t))^{-1}(I-B(t)) \\
\left.\quad \times\left(I-\left(I-B^{*}(t)\right)(I+W(t))^{-1}\right)(I-B(t))\right] Y(t+1) \\
=Y^{*}(t+1)\left[I+W(t+1)-\left(I-B^{*}(t)\right)(I+W(t))^{-1}(I-B(t))\right] Y(t+1) .
\end{gathered}
$$

From(1.12)-(1.14) we know that if $t$ is sufficiently large, we have $W(t)<0$, i.e., $0<I+W(t)<I$. Furthermore,

$$
\begin{aligned}
-(I & -B(t))^{*}(I+W(t))^{-1}(I-B(t))<-(I-B(t))^{*}(I-B(t)) \\
& =-I+\left(B^{*}(t)+B(t)\right)-B^{*}(t) B(t) .
\end{aligned}
$$

Combining (1.15), (1.16), and the condition, we see that

$$
\Delta\left(Y^{*}(t)(I-B(t)) Y(t+1)\right) \leq 0,
$$

i.e., $\left(Y^{*}(t)(I-B(t)) Y(t+1)\right)^{-1}$ is increasing. This contradicts (1.14) and completes the proof.

Next we consider two matrix systems:

$$
\begin{aligned}
& \Delta Y(t)=B_{i}(t) Y(t+1)+C_{i}(t) Z(t), \\
& \Delta Z(t)=-A_{i}(t) Y(t+1)-B_{i}^{*}(t) Z(t), \quad i=1,2 .
\end{aligned}
$$

We make the same assumption on $(1.17)_{i}$ as $(1.8)_{i}$. Using Theorem 3 , it is easy to show:

Theorem 9. If $(1.17)_{1}$ is nonoscillatory and $D_{2}(t) \geq D_{1}(t)$ for $t \geq t_{0} \geq M-1$ for some integer $t_{0}$, then $(1.17)_{2}$ is nonoscillatory as well.

Next we wish to consider certain subsystems of (1.2). To this end we denote $R=\left\{i_{1}, i_{2}, \ldots, i_{k}\right\}, 1 \leq i_{1}<i_{2}<\cdots<i_{k} \leq d, A(t)=\left(a_{i j}\right)_{d \times d}, B(t)=$ $\left(b_{i j}\right)_{d \times d}$, and $C^{-1}(t)=\left(c_{i j}\right)_{d \times d}$. We suppose that $B(t)$ satisfies $b_{i j}=0$ if $i \notin R$ and $j \in R$.

Let

$$
\widetilde{A}(t)=\left(\tilde{a}_{i j}\right)_{k \times k}, \quad \widetilde{B}(t)=\left(\tilde{b}_{i j}\right)_{k \times k}, \quad \tilde{C}(t)=\left(\tilde{c}_{i j}\right)_{k \times k}^{-1},
$$

where $\tilde{a}_{i j}=a_{l_{i} l_{j}}, \tilde{b}_{i j}=b_{l_{i} l_{j}}, \tilde{c}_{i j}=c_{l_{i} l_{j}}$ if $l_{i}, l_{j} \in R$.

For the $k \times k$ matrix system,

$$
\begin{aligned}
& \Delta \widetilde{Y}(t)=\widetilde{B}(t) \tilde{Y}(t+1)+\widetilde{C}(t) \tilde{Z}(t), \\
& \Delta \widetilde{Z}(t)=-\widetilde{A}(t) \tilde{Y}(t+1)-\widetilde{B}^{*}(t) \widetilde{Z}(t) .
\end{aligned}
$$


We have

Theorem 10. If (1.18) is oscillatory, so is (1.2).

Proof. Suppose (1.18) is oscillatory. Then by Theorem 1 we can find two integers $M, N$ such that there exists a nonzero vector sequence $\tilde{u}(t)=\left(\begin{array}{c}\tilde{y}(t) \\ \tilde{z}(t)\end{array}\right) \in \widetilde{\Omega}$, $\tilde{y}(t)=\left(\tilde{y}_{j}\right)_{k}, \tilde{z}(t)=\left(\tilde{z}_{i}\right)_{k}$, with $\tilde{q}[\tilde{u}] \leq 0$. (Here $\tilde{q}, \widetilde{\Omega}$ correspond to (1.18).)

Let

with

$$
u=\left[\begin{array}{l}
y(t) \\
z(t)
\end{array}\right], \quad y(t)=\left(y_{j}\right)_{d}, \quad z(t)=\left(z_{j}\right)_{d},
$$

$$
y_{i_{j}}=\left\{\begin{array}{ll}
\tilde{y}_{j} & \text { if } i_{j} \in R, \\
0 & \text { otherwise },
\end{array} \quad z_{i_{j}}= \begin{cases}\tilde{z}_{j} & \text { if } i_{j} \in R, \\
0 & \text { otherwise },\end{cases}\right.
$$

Then from $q[u]=\tilde{q}[\tilde{u}] \leq 0$ we conclude that $(1.2)$ is oscillatory.

Remark. If $B(t) \equiv 0$, then Theorem 10 establishes the conjecture of [7].

Theorem 11. If $C^{-1}(t) \leq M$ (constant Hermitian matrix), $\sum_{t=M}^{\infty} h(t)$ exists, and (1.2) is nonoscillatory, then

$$
\lim _{t \rightarrow \infty} C^{-1}(t) B(t)=L \quad(\text { constant Hermitian matrix }) ;
$$

furthermore, $L \leq M$.

Proof. Since (1.2) is nonoscillatory, there exists a Hermitian solution $W(t)$ for $t \geq t_{0}$ (some integer $t_{0} \geq M$ ) of (1.3) with $W(t)+C^{-1}(t)>0$.

Taking the summation of both sides of (1.3), we have

$$
-W(t+1)=W\left(t_{0}\right)+\sum_{\tau=t_{0}}^{t} \rho(\tau)+\sum_{\tau=t_{0}}^{t} h(\tau) .
$$

Now since $-W(t+1) \leq C^{-1}(t+1) \leq M$, and since $\sum_{\tau=t_{0}}^{t} h(\tau)$ exists, it follows that $\sum_{\tau=t_{0}}^{\infty} \rho(\tau)$ exists, so $\lim _{t \rightarrow \infty} W(t)=W_{0}$, a constant Hermitian matrix with $W_{0} \geq-M$.

Since $\rho(t) \geq 0$, we have $\lim _{t \rightarrow \infty} \rho(t)=0$. From (1.19) and $C^{-1}(t) \leq M$, it follows that $W(t)$ is bounded, i.e., $\lambda_{d}\left(\left(C^{-1}(t)+W(t)\right)^{-1}\right)$ does not go to zero as $t \rightarrow \infty$.

From the Courant-Fisher Theorem [6] we get

$\lambda_{1}(\rho(t)) \geq \lambda_{1}\left[\left(W(t)+C^{-1}(t) B(t)\right)^{*}\left(W(t)+C^{-1}(t) B(t)\right)\right] \lambda_{d}\left[\left(C^{-1}(t)+W(t)\right)^{-1}\right]$.

Now let $t \rightarrow \infty$ to obtain

$$
\lim _{t \rightarrow \infty} \lambda_{1}\left(\left(W(t)+C^{-1}(t) B(t)\right)^{*}\left(W(t)+C^{-1}(t) B(t)\right)\right)=0,
$$

i.e.,

$$
\lim _{t \rightarrow \infty}\left(W(t)+C^{-1}(t) B(t)\right)=0
$$

i.e.,

This completes the proof.

$$
\lim _{t \rightarrow \infty} C^{-1}(t) B(t)=\lim _{t \rightarrow \infty}(-W(t))=-W_{0} \leq M .
$$

Corollary 12. Suppose $C^{-1}(t) \leq M$ (constant Hermitian matrix) and $\liminf _{t \rightarrow \infty} \sum_{\tau=M}^{t} \lambda_{d}(h(\tau))>-\infty$. Then there exists a Hermitian solution $W(t)$ for $t \geq t_{0} \geq M$ of (1.3) which satisfies $\lim _{t \rightarrow \infty}\left(W(t)+C^{-1}(t) B(t)\right)=0$; furthermore, $C^{-1}(t) B(t)$ is bounded. 


\section{REFERENCES}

1. Calvin D. Ahlbrandt, Dominant and recessive solutions of symmetric three term recurrences, J. Differential Equations (to appear).

2. W. Coppel, Disconjugacy, Lecture Notes in Math., vol. 220, Springer, New York, 1971.

3. L. Erbe and P. Yan, Weighted averaging techniques in oscillation theory for second order difference equations, Canad. Math. Bull. 35 (1992), 61-69.

4. __ Disconjugacy for linear Hamiltonian difference systems, J. Math. Anal. Appl. 167 (1992), 355-367.

5. __, Qualitative properties of Hamiltonian difference systems, J. Math. Anal. Appl. (to appear).

6. P. Lancaster and M. Tismenetsky, The theory of matrices, Academic Press, New York, 1985, pp. 286-289.

7. A. Peterson and J. Ridenhour, Oscillation of second order linear matrix difference equations, J. Differential Equations 89 (1991), 69-88.

Department of Mathematics, University of Alberta, Edmonton, Alberta, Canada T6G 2G1 